\title{
Strongyloides stercoralis Hyper Infection in an Immunocompromised Patient a Case Report
}

\author{
Biswajeet Sahoo ${ }^{1}$, Sanjeeb Sharma ${ }^{1}$, Thokchom Devaraj Singh ${ }^{1}, \mathrm{H}$. \\ Rebachandra Singh ${ }^{2}$,Waikhom Shashi Singh ${ }^{1}$, Sudipta Naorem ${ }^{1}$. \\ ${ }^{1}$ Post graduate student, Department of Microbiology, Regional Institute of Medical Sciences, \\ Imphal, Manipur, India. \\ ${ }^{2}$ Associate Professor, Department of Microbiology.
}

\begin{abstract}
:
Background: Strongyloidiasis is an intestinal parasitic disease caused by the nematode Strongyloides stercoralis and is common in tropical countries. In immunocompromised hosts, the overwhelming accelerated auto infective cycle can potentially lead to a life-threatening illness with multi-organ failure due to a massive larval invasion known as hyper infection syndrome.

Case Presentation: A 46 years old female farmer (the patient was immunocompromised as she took high dose steroids for about four years as treatment for rheumatoid arthritis) was admitted at Regional Institute of Medical Sciences, Imphal, hospital in the Department of Medicine with chief complaints of swelling of limbs for 3 months and intermittent pain in the abdomen ( colicky in nature) for one month, with nausea, vomiting and dark coloured watery diarrhoea for 10 days. Her stool was examined and a large number of rhabditiform larvae of Strongyloides stercoralis were seen in stool wet mount microscopy. She was treated with subcutaneous ivermectin.
\end{abstract}

Conclusion: Hyperinfection by Strongyloides stercoralis was found in this case which is a common finding in immunocompromised patients. Early diagnosis and aggressive treatment with subcutaneous ivermectin should be done to save the life of the patient.

Keywords: hyperinfection, immunocompromised, autoinfection

\section{Introduction}

Strongyloides stercoralis is a soil-transmitted intestinal nematode that infects people, especially in tropical and subtropical regions ${ }^{(1)}$. The usual route of transmission is by penetration of the skin by filariform larvae (infective form), following contact with contaminated soil ${ }^{(1)}$. The results of several studies have documented an association of disseminated Strongyloides infection with malignant tumors, severe malnutrition, prolonged steroid therapy and renal transplantation ${ }^{(2)}$.

In immunocompetent hosts, the infection commonly leads to minor symptoms such as transient diarrhoea and abdominal pain, and may be asymptomatic and latent for decades ${ }^{(3)}$. In immunocompromised hosts, the overwhelming accelerated autoinfective cycle can potentially lead to a life-threatening illness with multi-organ failure due to a massive larval invasion known as hyperinfection syndrome ${ }^{(3)}$.

Hyperinfection syndrome is not exactly defined, but the hallmark is an increase in the number of larvae in the stool and/or sputum along with the manifestations confined to respiratory and gastrointestinal systems ${ }^{(4)}$.Hyperinfection syndrome is estimated to happen in 1.5 to $2.5 \%$ of the patients with strongyloidiasis ${ }^{(5)}$.

The reported mortality rate of this hyperinfection syndrome is high (up to 87\%), highlighting the importance of prophylactic anthelminthic treatment in immunocompromised patients ${ }^{(6)}$. The intestinal ileus associated with the hyperinfection syndrome can reduce the bioavailability and the efficacy of an oral formulation of anti parasitic drugs and should also be treated with subcutaneous ivermectin ${ }^{(3)}$.

\section{Case Report}

A 46 years old female, farmer by profession from a rural area of Imphal was admitted in the Department of Medicine, Regional Institute of Medical Sciences, Imphal, hospital with chief complaints of swelling of limbs for 3 months and intermittent pain in the abdomen( colicky in nature) for one month and also complained of nausea, vomiting, breathlessness and dark coloured watery diarrhea for 10 days. As per the patient, bladder, bowel and sleep pattern were normal. The patient complained of loss of appetite for about four months before admission. There was past history of prolonged high dose corticosteroid therapy for 4 years for the treatment of rheumatoid arthritis as per the patient after thorough evaluation.

On clinical examination, her BP was low with a value of $60 / 90 \mathrm{~mm} \mathrm{Hg}$, she had pallor, edema especially over the lower limbs and looked severely malnourished. All the laboratory investigation done on the 
day of admission were found normal except for low level of total serum protein $(3.5 \mathrm{gm} \%) \&$ serum albumin (1.2 gm\%);low Hb level of (10 gm\%), and eosinophilia (12\%). Patient is neither hypertensive nor diabetic and has no history of tuberculosis as well as is found negative for hepatitis B, C and HIV.

Stool R/E was done on the third day of admission and it showed a large number of Strongyloides stercoralis rhabditiform larva ( $\geq 35$ larvae per ocular field) Fig-1. Fresh stool was taken and $25 \mathrm{gm}$ of it was processed by adopting modified Baermann funnel technique ${ }^{(4)}$ for further observation (using a stereoscopic dissecting binocular microscope) and morphotaxonomical studies. The larva when observed under a compound microscope having a high magnifying power (Nikon, Eclipse E-200), was found to have possess features like shallow buccal cavity, short oesophagus, attenuated tail ${ }^{(5)}$ and a distinct easily visible genital rudiment. All these characters/morphological features are the taxonomically important and valid diagnostic features for systematic study of the rhabditiform larvae of Strongyloides stercoralis. Thus, based on the presence of these taxonomically valid characters, the larva has been identified as rhabditiform larva of S.stercoralis.

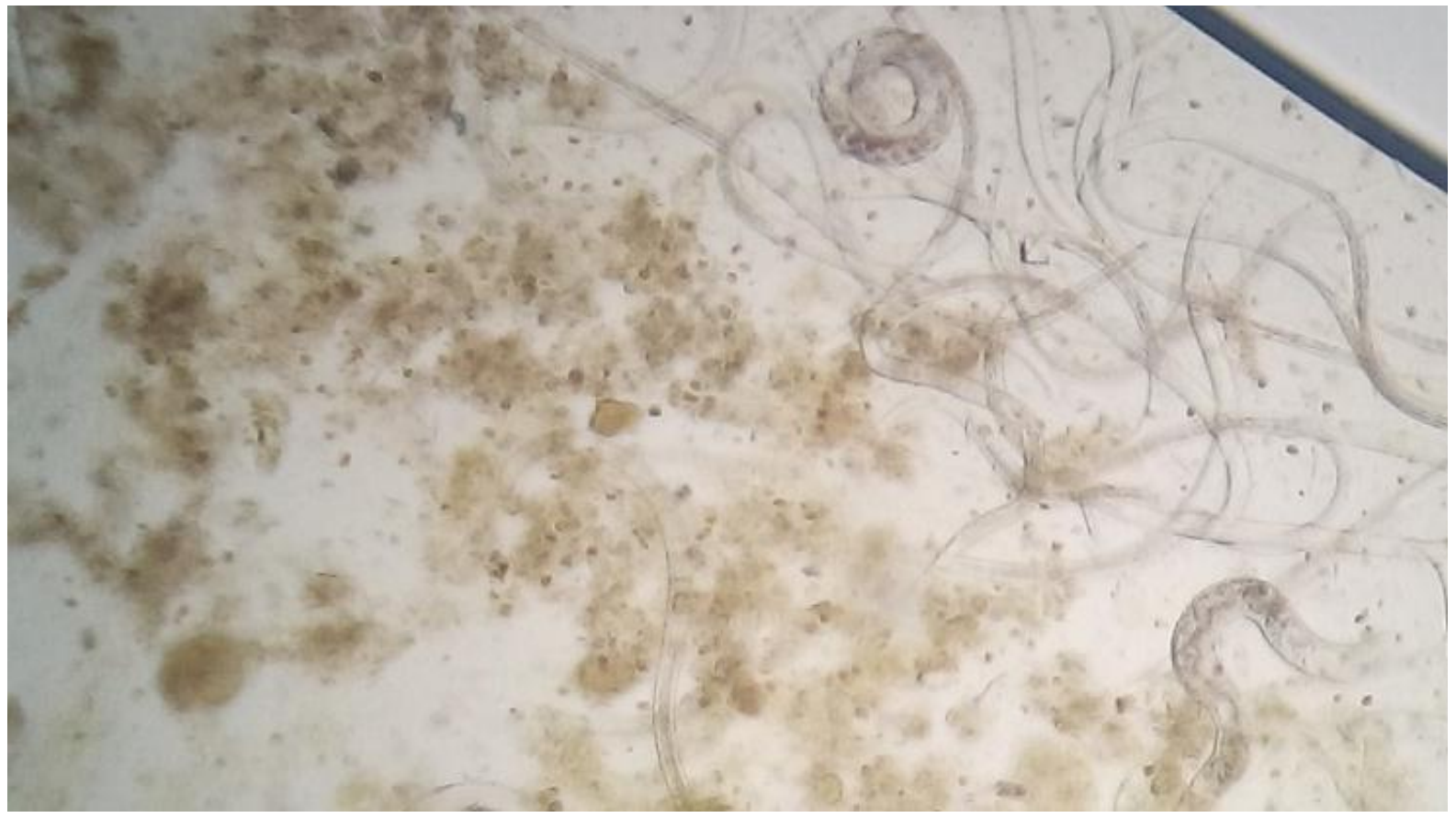

Fig-1- Showing large no fo Strongylodes stercoralis in the stool under compound microscope

Stool culture was also done using agar plate method $^{(6)}$ and presence of strongyloides larvae was observed. Sputum examination showed no strongyloides larva.

On the day of admission the patient was treated with i.v fluids and symptomatic supportive therapy. On second day she got blood transfusion and albumin injection and was continued for third day with second blood unit and 400mg of oral albendazole therapy. Administration of protein powder was started on fourth day. Inspite of treatment, patient condition became more serious with increased pain in the abdomen and generalized weakness. After the stool examination report, $15 \mathrm{mg}$ of ivermectin once daily was started from fifth day onwards but she started having difficulty in respiration from next day which lead to respiratory failure after few days.

\section{Discussion}

Strongyloides stercoralis are found in warm and humid soil. Filariform larvae infect humans through the skin of bare feet. After infection, larvae migrate to the duodenum and grow into mature females. Rhabditiform larvae hatched from eggs are excreted from the host along with faeces. However, some develop into filariform larvae and reinfect through the colon or anal skin (autoinfection). Once infected, the infected individual remains in a carrier state for a long time ${ }^{(7)}$.

Strongyloides stercoralis hyperinfection syndrome has been described in immunocompromised patients. The most common predisposing factors are the use of prolonged and high dose steroids and HTLV-I infection ${ }^{(8)}$. Other underlying diseases leading to immunodeficiency such as lymphomas, leukemias, or infection with human immunodeficiency virus have been reported to facilitate Strongyloides hyperinfection ${ }^{(9)}$. In hyperinfection and dissemination, complete disruption of the mucosal patterns, ulcerations, and paralytic 
ileus have been observed. Bacterial and fungal infections often occur in cases of hyperinfection because of the leakage of gut flora from a bowel damaged by moving larvae ${ }^{(10)}$.

Ivermectin is now recognized as the drug of choice for treating disseminated strongyloidiasis, compared to thiabendazole and albendazole, as it shows comparable and better rates of larval clearance and fewer and comparable side effects than the latter two drugs ${ }^{(11)}$. However, since the clinical features of Strongyloides stercoralis hyperinfection include intestinal ileus, oral absorption of the drugs may be impaired $(12)$.

Although the gastrointestinal tract is the primary site of Strongyloides infection, associated symptoms such as abdominal pain and intermittent diarrhoea may be vague; if ignored by the clinician, lead to fatal dissemination and clinical deterioration of the patient like in our case. Eliciting an appropriate occupational and drug history from patients are the essential epidemiological clues for establishing early diagnosis.

As the stool sample of the patient was delayed in sending to our laboratory, diagnosis was delayed and hence despite immediately starting subcutaneous ivermectin after our reporting the patient died with respiratory failure after 3 days of treatment.

\section{Conclusion}

On the basis of our finding, we recommend early routine testing of patients for stool examination especially for patients who are residing from rural areas and engaged in farming and Strongyloides hyper infection should be kept as a differential and highly suspected in an immunosupressed patient with abdominal pain presenting with watery diarrhea, generalized edema, eosinophillia and low protein levels and this should be followed by early intensive treatment with subcutaneous ivermectin.

\section{References}

[1]. Eduardo G, Angelica T, Humberto A, Raul T, Rosa I, Douglas MW, Strongyloides stercolaris hyperinfection associated with human T cell lymphotropic virus type -1 infection in Peru, Am J Trop Med Hyg, 60(1), 1999, 146-149.

[2]. Concha R, Harrington WJ, Rojers Al, Intestinal strongyloidiasis: recognation, management and determinants of outcome. J Clin Gastroentero, 39, 2005, 203-11.

[3]. Jerome P, Marie DS, Antoine R, Christine L, Jacques G, Veronique L, Subcutaneous Ivermectin as a safe salvage therapy in Strongyloides stercolaris hyperinfection syndrome: A case report, Am J Trop Med Hyg, 73(1), 2005, 122-124.

[4]. Schimdt ,G. D. (1992): Essentials of Parasitilogy, $4^{\text {th }}$ Edition ( $2^{\text {nd }}$ Indian Reprint), Universal Book Stall, New Delhi- P- 259-60.

[5]. WHO (1994):Bench Aids for the diagnosis of intestinal parasites, plate -2, Helminths, Reprinted 2003. Denham, D. A. and Suswillo (1995): Diagnosis of intestinal Helminth infections in medical parasitology. A practical Approach,Edited by Gillespie, S.H. and Hawkey,P.M, Reprinted 2002, Oxford University Press, P-257 and p-262-63. WHO (2003): Manual of Basic Technique for a health laboratory, $2^{\text {nd }}$ Edition, Geneva, ISBN no. 9241545305,PP-156-158.

[6]. John, D. T. and Petri Jr.W.A. (2009): In Markell and Voges Medical Parasitology, 9 $^{\text {th }}$ edition, Elsevier, Chapter-14 (examination of stool specimen), P-408. Gillespie, S. H. (1995): Migrating worms, In Medical Parasitology, A practical approach. Edited by Gillespie, S. H. And Hawkey, P. M., reprinted 2002, Oxford University Press, p-185.

[7]. Tetsuo H, Nobufumi U, Kazuto K, Osamu Z, Nagisa K, Akira H, Impairment of host immune response against Strongyloides stercolaris by human T cell lymphotropic virus type linfection, Am J Trop Med Hyg, 74(2), 2006, 246-249.

[8]. Cruz T, Reboucas G, Rocha H, Fatal strongyloidiasis in patients receiving corticosteroids, N Engl J Med 275, 1966, $1093-1096$.

[9]. Celedon JC, Mathur Wagh U, Fox J, Garcia R, Systemic strongyloidiasis in patients infected with the human immunodeficiency virus. A report of 3 cases and review of the literature, Medicine, 73, 1994, 256-263.

[10]. Afzal A Siddiqui, Steven L Berk, Diagnosis of Strongyloides stercoralis Infection, Clinical Infectious diseases, 33(7), 2001, 104047.

[11]. Gann PH, Neva FA, Gam AA, A randomized trial of single- and two-dose ivermectin versus thiabendazole for treatment of strongyloidiasis, J Infect Dis 169, 1994, 1076-1079.

[12]. Boken DJ, Leoni PA, Preheim LC, Treatment of Strongyloides stercoralis hyperinfection syndrome with thiabendazole administered per rectum, Clin Infect Dis 16, 1993, 123-126. 